\title{
Research and Simulation of fault prediction method in numerical control machine tool operation
}

\author{
Chen Jinxia \\ Inner Mongolia Technical College Of Mechanics And Electrics \\ 010070
}

Keywords: numerical control machine tool; fault diagnosis; firefly algorithm

\begin{abstract}
: in the studying process of fault prediction method during operating numerical control (NC) machine tool, using the current algorithm to predict fault in the running process of the NC machine tool, iteration is easy to fall into the local extremum problem. Therefore, a new method of fault prediction based on the improved firefly algorithm for NC machine tool is proposed. First, the firefly algorithm is used to analyze the running process of a NC machine tool, and NC machine tool fault diagnosis model is established. Then, the background value optimization and NC machine tool fault diagnosis model are combined and applied in NC machine tools fault prediction, in the predicting process, the effect of background value and initial conditions on the fault prediction accuracy of the operation process of the NC machine tool, using iterative process to update the original data, and the particle swarm optimization algorithm is fused to optimize the background value of each iteration, in the end, NC machine tool fault prediction can be completed effectively. The simulation results prove that the method of fault prediction of the NC machine tool based on the improved firefly algorithm has high accuracy and robustness.
\end{abstract}

\section{Introduction}

Numerical control (NC) machine tool is an important index of measuring the level of mechanical manufacturing process ${ }^{[1-3]}$. With the development of modern science and technology, the requirements for availability of NC machine tools is increasingly high ${ }^{[4]}$. However, the structure of NC machine tools is more complex, there are more parts, and the probability of failure is higher ${ }^{[5-7]}$. How to reduce the probability of the failure occurrence of NC machine tools, has become a prominent problem throughout the whole life cycle of NC machine tool. Fault prediction method is an effective way to solve this problem, which has caused many experts and scholars' attention ${ }^{[8-10]}$.

At present, the main method of fault prediction is based on genetic algorithm, ant colony algorithm and wavelet algorithm. Among them, the fault prediction method based on wavelet algorithm is commonly used, but there is a problem of this algorithm, it is easy to fall into local extremum.

In view of the above problems, the fault prediction method based on the improved firefly algorithm of NC machine tool is put forward. The method has high accuracy and robustness.

\section{$\mathbf{2}$ fault prediction principle in the operation process of NC machine tools}

In the operation process of a NC machine tool fault prediction, first to divide spindle drive system of NC machine tools according to the fault level, parameter level, feature level and state level hierarchically, for the NC machine tool failure history, based on the viewpoint of whole life cycle to establish machining center failure rate distribution curve includes the early failure period. The fault occurrence time is regarded as limitation, according to the NC machine tool fault evolution rate to determine the critical time point by using the method of higher mathematics, and then complete NC machine tool fault prediction effectively. Specific steps are as follows:

During the fault prediction process of the NC machine tool in running process, select the fault in the operation process of the $t$-th NC machine tools as data set $p_{t}, t \in[1, p]$ of foreboding parameters within a certain time $T$, according to the equal interval to divide into $E^{(P t)}$ parts, and $N^{(P t)}$ sampling values are selected from each interval.

$$
p_{t}=\left\{x_{i, j}\right\} i \in\left[1, E^{(P t)}\right] j \in\left[1, N^{(P t)}\right]
$$

In the fault prediction of the NC machine tool during running process, the sampling value within 
each interval is defined to correspond to a foreboding state of NC machine tool fault $S_{i}^{(p t)}$, so as to obtain foreboding state sequence of NC machine tool fault during the running process of the NC machine tools:

$$
\left\{S_{i}^{(p t)} \mid i \in\left[1, E^{(p t)}\right]\right\}
$$

\section{3 fault prediction optimization principle in the operation process of NC machine tools}

\section{1 optimized construction of fault diagnosis model of NC machine tool}

In the fault optimization prediction of NC machine tool during running process and fused with the firefly algorithm, using formula (3) to calculate the objective function in the failure prediction of $\mathrm{NC}$ machine tool during running process, and the results is regarded as initial value in NC machine tool fault decision range during the running process:

$$
r_{d}^{i}(k+1)=\min \left\{r_{q}, \max \left[0, r_{d}^{i}(k)+\beta\left(m_{i}-\left|M_{i}(k)\right|\right)\right]\right\}
$$

In the above formula, fault decision range of firefly $i$ in the $k$ iterations is represented by the $r_{d}^{i}(k)$ and meet the condition $0 \leq r_{d}^{i} \leq r_{q}, r_{q}$ is the sensing range of the firefly, $m_{i}$ is control threshold for the number of firefly neighbours, $\beta$ represents the change rate of fault optimization prediction fields of the NC machine tool in the running process, $M_{i}(k)$ is the neighbor set of firefly $i$ in $k$ iterations.

In the fault optimized prediction of NC machine tool during running process, after repeated iteration, the fault optimization prediction data of NC machine tool in the operation process is output, the individual fitness value $D$ is viewed as the sum of the absolute value of the error between fault optimization prediction output and expected output, and expressed by the follow equation:

$$
D=k\left(\sum_{i=1}^{n}\left|o_{i}-y_{i}\right|\right)
$$

In the fault optimized prediction of NC machine tool during running process, the fault diagnosis model can be set up by the use of the following equation:

$$
l_{i}(k)=(1-\lambda) l_{i}(k-1)+\omega f\left(x_{i}(k)\right)
$$

\section{2 fault prediction optimization principle in the operation of NC machine tools}

In the fault optimized prediction of NC machine tool during running process, the simulation fault prediction value of the original sequence represented by ${ }^{(0)}(k)$, set up the following equations:

$$
\left\{\begin{array}{l}
\frac{\partial E}{\partial a}=0 \\
\frac{\partial E}{\partial a}=0
\end{array}\right.
$$

In the fault optimized prediction of NC machine tool during running process, based on the above formula to repeatedly minus (difference) for $\tilde{x}^{(0)}(k)$, fault optimization stimulation prediction value in the original sequence can be obtained:

$\tilde{x}^{(0)}(k)=\tilde{x}^{(1)}(k+1)-\tilde{x}^{(1)}(k)$

$p=\{0.5\}$ as the fault prediction background value vector, the different faults background prediction value $p$ has different effect on fault prediction optimization model accuracy, seeking optimal fault background prediction values and initial conditions, and combining the fault prediction background value optimization and NC machine tool fault diagnosis model and applying to NC machine tools fault prediction, under the corresponding NC machine tool fault diagnosis model test criteria, using the following equation to minimize the error of fault optimization prediction of NC machine tool during the operation process: 
$e(k)=\left|\frac{\tilde{x}^{(0)}(k)-x^{(0)}(k)}{\tilde{x}^{(0)}(k)}\right|$

In fault optimized prediction, using an iterative process to update the original fault data and predict the next NC machine tool failure data (the $m+1$ data), and then in the original series, remove the first NC machine fault data, add a new NC machine tool failure data, continue to build the model to predict the next NC machine tool failure data, by analogy, each time remove an old NC machine tool failure data and add a new data, and then use the new NC machine tool failure data group re-estimate parameters $a, b$, this constantly cycle, so as to continue to carry out iterative for initial sequence, expressed by:

$$
\tilde{x}^{(1)}(k+1)=\left[\tilde{x}^{(1)}(1)-\frac{b}{a}\right] e^{-a k}+\frac{b}{a}
$$

In the fault optimized prediction of NC machine tool during running process, the particle swarm optimization algorithm is used to optimize the fault prediction background value of each iteration. The specific steps are as follows:

(1) In the fault optimized prediction of NC machine tool during running process, each particle's fitness and fitness of experienced global best position gbest are compared, if the former is superior to the latter, the former location will be regarded as the global optimum.

(2) In the fault optimized prediction of NC machine tool during running process, after collecting the following two failures optimal prediction extremum, according to the speed and location evolution equation as follows to update the particle:

$$
V_{i}(j+1)=k\left[w V_{i}(j)+c_{1} r_{1}\left(x_{i}^{\text {pbest }}(j)-X_{i}(j)\right)+c_{2} r_{2}\left(x_{i}^{\text {pbest }}(j)-X_{i}(j)\right)\right]
$$

(3) In the fault optimized prediction of NC machine tool during running process, the solution is output if the termination condition is satisfied, otherwise returned to (1).

\section{4 experiments and simulation}

In order to prove the validity of the fault prediction method based on the improved firefly algorithm for the NC machine tool, a test is needed. The improved algorithm prediction model and the traditional algorithm prediction model are applied to the fault prediction of the spindle of a NC lathe. In the test, failure data of seven fields were collected, failure time is $\{117.99,201.39,292.13,400.81,526.50,688.97,899.03\}$, predicted results of different algorithms model and the original fault data values were compared, and used to measure the effectiveness of different algorithms for fault prediction.

Table 1 Comparison of fault prediction results of different algorithms

As can be seen from table 1, the prediction result of the improved algorithm is better than the

\begin{tabular}{ccccc}
\hline NO. & Original fault data & $\begin{array}{c}\text { Predicted data of using } \\
\text { improved algorithm }\end{array}$ & $\begin{array}{c}\text { Predicted data of using } \\
\text { improved algorithm }\end{array}$ & $\begin{array}{r}\text { trad } \\
\text { itio } \\
\text { nal }\end{array}$ \\
1 & 117.99 & 117.96 & 129.96 & alg \\
2 & 201.39 & 201.29 & 301.29 & orit \\
3 & 292.13 & 292.12 & 296.12 & hm, \\
4 & 400.81 & 400.81 & 404.81 & the \\
5 & 526.50 & 526.50 & 529.50 & pre \\
6 & 688.97 & 688.97 & 689.97 & dict \\
7 & 899.03 & 899.03 & 999.03 & ion
\end{tabular}

accuracy is higher, and the robustness is better. This is because the improved algorithm considering the effect of background value and initial conditions to the NC machine tool failure prediction accuracy, using an iterative process to update the original data, and the background value of each iteration is optimized.

\section{Conclusions}

In view of the current algorithm to predict fault in the running process of the NC machine tool, 
iteration is easy to fall into the local extremum problem. Therefore, a new method of fault prediction based on the improved firefly algorithm for NC machine tool is proposed. First, the firefly algorithm is used to analyze the running process of a NC machine tool, and NC machine tool fault diagnosis model is established. Then, the background value optimization and NC machine tool fault diagnosis model are combined and applied in NC machine tools fault prediction, in the predicting process, the effect of background value and initial conditions on the fault prediction accuracy of the operation process of the NC machine tool, using iterative process to update the original data, and the particle swarm optimization algorithm is fused to optimize the background value of each iteration, in the end, NC machine tool fault prediction can be completed effectively. The simulation results prove that the method of fault prediction of the NC machine tool based on the improved firefly algorithm has high accuracy and robustness.

\section{References}

[1] Chen Quanming, Gao Xu, Lu Xiangsheng. Repair method for the failure in CNC machine tool running [J]. China Science and Technology Expo, 2013. (26): 18-18.

[2] Yang Dongsheng, Li Hongwei, Sun Yilan, et al. Study on Novel Grey Relation Analysis Method and Its Application in Fault Recognition of NC Machine Tool Spindle [J]. China Mechanical Engineering, 2013, 24 (23): 3150-3153.

[3] Li Yong, Xue Mei. Exploration of Modern Vocational Education Methods in CNC Machine Fault Diagnosis and Repair Course [J]. The guide of science and education 2013, (31):170-170.

[4] Zhang Huan, Zhang Xinyu, Huang Haifeng. Research on fault prediction of the spindle system of CNC machine tool [J]. Management \& Technology of SME, 2014, (9): 238-238.

[5] Shi Xinwei. Analysis of the failure of CNC machine tools and processing methods [J]. Chinese e-commerce, 2013, (23): 71-71.

[6] Meng Yongmei. Study on the problems and countermeasures of CNC machine tool repair and renovation [J]. Science and technology innovation and application, 2014, (3): 83-83.

[7] Li Qinsheng, Wei Ping, Liu Xinyu. Research \& Application of Post-processing and Machining Simulation in NC Machine Tool [J]. Mechanical engineer, 2013, (10): 130-132.

[8] Huang Mingguo, Gong Dongtao, Huang Anlian. Application and troubleshooting of PLC in numerical control machine tools [J]. China new telecommunications, 2013, (11): 47-47.

[9] Ji Xiaoming. Failure process and research of the CNC machine toolchanger in the manufacturing system () [J]. Commodity and quality: Academic observation, 2013, (1): 99-99.

[10] Huang Bo, Ding Hao, Zhang Xiaofang, et al. Predication Method of Complex Equipment Based on RBF Neural Network [J]. Computer simulation, 2014, 31 (1): 14-17. 